\title{
Effect of Properties of Phosphate Buffer Saline Solutions on Vibrational Characteristics of a Quartz Crystal Microbalance Resonator
}

\author{
Jinxing Liang, ${ }^{1 *}$ Xi Zhao, ${ }^{1}$ Debo Kong, ${ }^{1}$ Yijing Chen, ${ }^{1}$ and Toshitsugu Ueda ${ }^{2}$ \\ ${ }^{1}$ Key Laboratory of Micro-Inertial Instrument and Advanced Navigation Technology, \\ Ministry of Education, School of Instrument Science and Engineering, Southeast University, \\ 2 Sipailou, Xuanwuqu, Nanjing 210096, China \\ ${ }^{2}$ Research Center of Graduate School of Information, Production and System, Waseda University, \\ Kitakyushu, Fukuoka 808-0135, Japan
}

(Received February 26, 2018; accepted April 19, 2018)

Keywords: QCM, pH value, conductivity, quality factor, conductance

Buffer type, concentration, and $\mathrm{pH}$ value are important factors that may affect protein formulation, stability, bioreactivity, and other behaviors. When biosensing application factors are the issues, the effect of buffer liquidity on the performance of the sensor should be noted. In this work, we investigated the effect of the phosphate buffer (PB) solution on the vibrational characteristics of a high-frequency quartz crystal microbalance (QCM) with an inverted-mesa structure using solutions with various concentrations and $\mathrm{pH}$ values. Experimental results show that increasing the $\mathrm{PB}$ concentration and $\mathrm{pH}$ value and adding $\mathrm{NaCl}$ decreased the resonant frequency and quality factor. Hence, in selecting the buffer, one should not only consider the bioissues, but also the effect on the sensor when a QCM is used as the transducer.

\section{Introduction}

$\mathrm{pH}$ buffers are commonly used in biochemistry to control $\mathrm{pH}$ and preserve protein stability. They are usually aqueous solutions of a weak acid (base) and its conjugated base (acid). ${ }^{(1)}$ Salts are usually added to $\mathrm{pH}$ buffers to increase the ionic strength and thereby enhance buffering effectiveness. It has been widely reported that buffers not only control $\mathrm{pH}$, but also have specific effects on protein activity, such as enzymatic catalysis, ${ }^{(2,3)}$ protein conformational stability, ${ }^{(4)}$ bioreactivity, ${ }^{(5-8)}$ and protein thermal events. ${ }^{(9)}$ The selection and optimization of buffer solutions are important issues to maintain proper bioactivity; typical factors include buffer type, buffer concentration, and identity of added salt and its concentration as described in the cited references. In biosensing systems, the buffer solution is usually used to establish a baseline for identifying signals produced by a bioreaction. In some cases, the effect of the buffer solution on the transducer may not be neglected. Weng et al. investigated the effect of the concentration of a phosphate buffer (PB) solution on an ion-sensitive field-effect transistor (ISFET)

*Corresponding author: e-mail: j-liang@seu.edu.cn http://dx.doi.org/10.18494/SAM.2018.1936 
and concluded that the concentration of PB solution must be carefully determined to achieve accurate results. ${ }^{(10)}$ Volotovsky et al. reported the effect of a reduction in PB concentration on the response of a glucose-sensitive ISFET-based biosensor. ${ }^{(11)}$

A quartz crystal microbalance (QCM) is a widely known biosensing tool developed using the famous mass frequency shift effect suggested by Sauerbrey in 1959. ${ }^{(12)}$ When used in a liquid, the resonant frequency of the QCM is affected by the liquid's mechanical properties (density and viscosity). ${ }^{(13)}$ In fact, the resonance of the QCM is also affected by the liquid's electrical properties. Nomura and Yanagihara reported the dependence of resonant frequency of electrode-separated QCMs on the conductivity and permittivity of the liquid. ${ }^{(14)}$ Bao et al. reported the $\mathrm{pH}$ dependence of the oscillation frequency of one-electrode-separated piezoelectric quartz crystals. ${ }^{(15)}$ Recently, with the development of the lateral field excitation (LFE) quartz crystal resonator, more works have been reported on the higher conductivity or permittivity sensitivity of LFE QCM than that of the conventional QCM. ${ }^{(16-19)}$

As already mentioned, when used in a liquid, it is clear that the resonating fundamental frequency of a QCM is affected by the liquid's electrical properties. In practical biosensor applications, the stability of the rosonant frequency is more important than the relative frequency shift caused by the liquid. However, until now, there has been no detailed report of the effect of the buffer solution on the oscillation characteristics of a QCM resonator. In this study, we experimentally investigate these effects by using a high-frequency QCM chip and a common $\mathrm{PB}$ solution at various $\mathrm{pH}$ values, concentrations, and added salt contents.

\section{Materials and Methods}

\subsection{Materials}

The high-frequency QCM chip used in this work was prepared using our well-established wet etching process. The QCM chip was designed with an inverted-mesa structure, and the vibration area is etched on one side. The excitation electrode on the etched side fully covers the etched area, which is larger than the electrode on the other side facing the liquid. The detailed fabrication process and chip characterization have been reported in previous studies from our laboratory. ${ }^{(20,21)}$ The dimensions of the QCM chip were 5 (length) $\times 5$ (width) $\times 0.1$ (thickness) $\mathrm{mm}^{3}$. The fundamental frequency was higher than $38 \mathrm{MHz}$, and the quality factor was larger than 20000 in air and 1000 in pure water.

Disodium hydrogen phosphate dodecahydrate $\left(\mathrm{Na}_{2} \mathrm{HPO}_{4} \cdot 12 \mathrm{H}_{2} \mathrm{O}, \mathrm{AR}\right)$ and sodium dihydrogen phosphate $\left(\mathrm{NaH}_{2} \mathrm{PO}_{4} \cdot 2 \mathrm{H}_{2} \mathrm{O}, \mathrm{AR}\right)$ were purchased from Nanjing Chemical Reagent Co., Ltd. and Shanghai Lingfeng Chemical Reagent Co., Ltd., respectively. Distilled water was employed as the solvent. The PB solutions were made from $0.2 \mathrm{M} \mathrm{Na}_{2} \mathrm{HPO}_{4}$ and $0.2 \mathrm{M}$ $\mathrm{NaH}_{2} \mathrm{PO}_{4}$ solutions. The variable concentrations $(0.01,0.05,0.1$, and $0.2 \mathrm{M})$ of $\mathrm{PB}$ solutions were made by diluting the original $\mathrm{Na}_{2} \mathrm{HPO}_{4}$ and $\mathrm{NaH}_{2} \mathrm{PO}_{4}$ solutions, and the $\mathrm{pH}$ values (5.8-8.0 at intervals of 0.2 ) were adjusted by mixing the diluted solutions at corresponding volume ratios. These selected buffer concentrations and $\mathrm{pH}$ values are commonly used in biosensing systems. 


\subsection{Measurement method}

The effect of PB solutions on the resonating characteristics was evaluated by measuring the admittance of the quartz crystal resonator. A flow injection system was established to introduce the PB solutions as reported in our previous report. ${ }^{(20)}$ The flow-injection-based measurement system consisted of a syringe pump, an injector, a tailor-made flow cell, and an impedance analyzer (Agilent 4294A). Distilled pure water was loaded into the syringe pump, and variable PB solutions were introduced through the injector. The QCM chip was housed in the flow cell, and the two excitation electrodes were connected to the impedance analyzer. The rosonant frequency was recorded at the maximum conductance. In addition, the $Q$ value and equivalent circuit parameters were recorded. The rosonant frequency in water was adopted as the fundamental frequency in the liquid. In this study, the main vibrational characteristics (rosonant frequency, $Q$ value, and conductance) were used to evaluate the effect of the liquid.

\section{Results and Discussion}

At first, the basic vibrational characteristics of the QCM chip were confirmed in air and in pure water as shown in Fig. 1. The $Q$ values were 22318 and 1080 in air and water, respectively. These results demonstrated that the system worked well.

The PB solutions were introduced into the flow cell using the injector. Figure 2(a) shows the measured dependence of QCM resonant frequency on the $\mathrm{pH}$ value and concentration of the buffer solution. The frequency shift was calculated by subtracting the resonant frequency in water from the measured resonant frequency in the PB solution. It can be clearly seen that the resonant frequency of the QCM gradually decreased as the $\mathrm{pH}$ value increased at a fixed concentration. The resonant frequency decreased as the concentration was increased at a

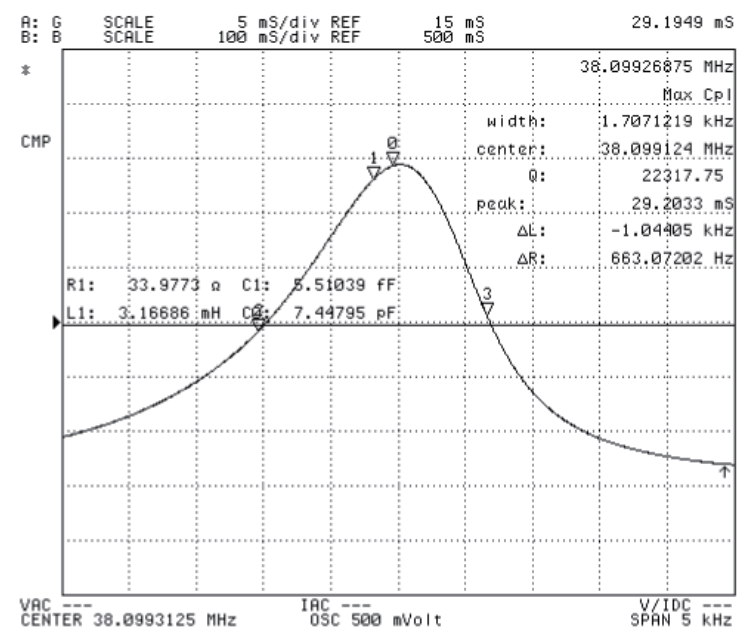

(a)

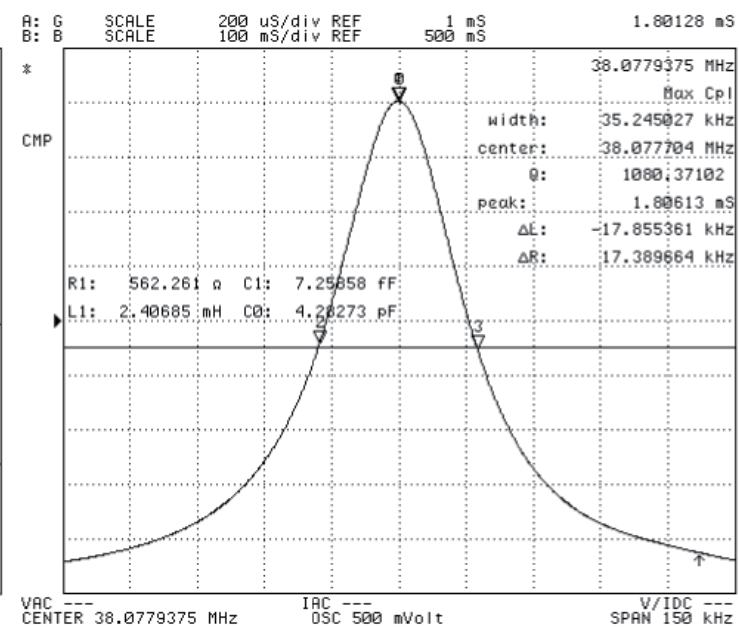

(b)

Fig. 1. Basic vibration characteristics of QCM chip (a) in air and (b) in water. 


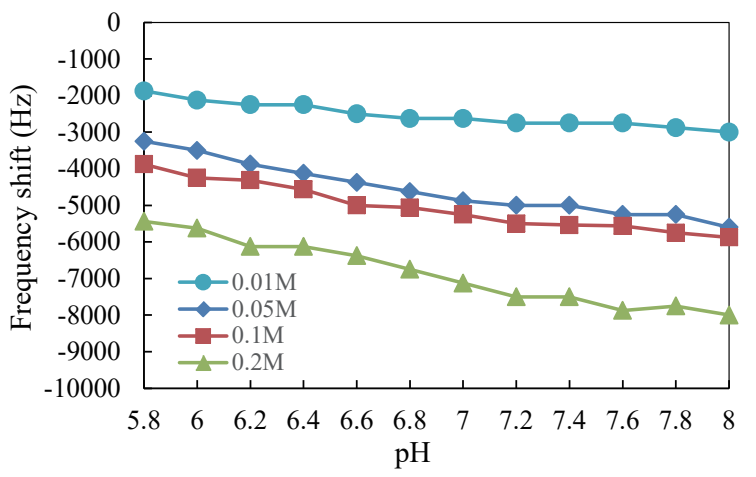

(a)

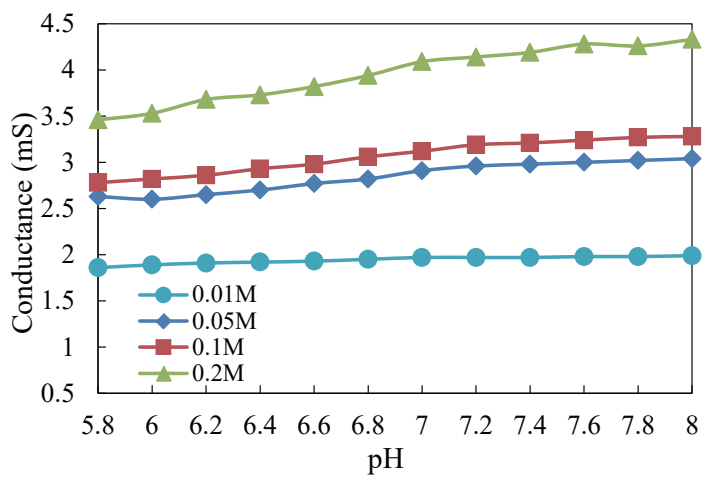

(b)

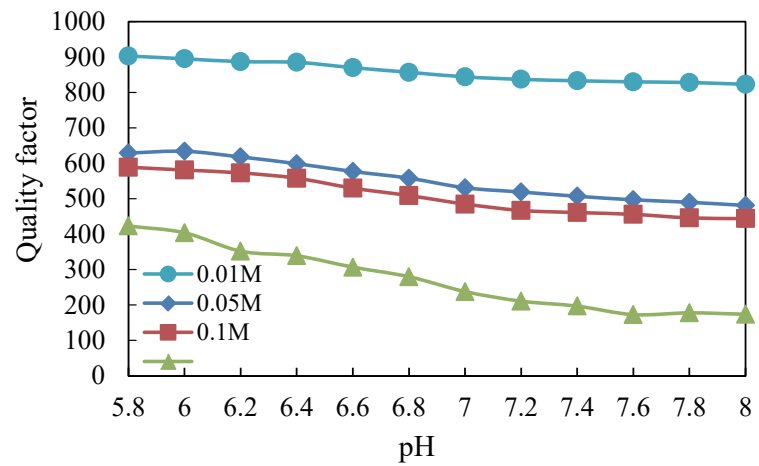

(c)

Fig. 2. (Color online) Dependence of resonant characteristics on buffer concentration and pH value: (a) resonant frequency, (b) conductance, and (c) $Q$ value.

constant $\mathrm{pH}$ value. Figure 2(b) shows the dependence of conductance on the PB concentration and $\mathrm{pH}$ value. The conductance increased gradually as the $\mathrm{pH}$ value was increased, and it increased even more as the concentration was increased. Figure 2(c) shows the effect of the PB solution on the quartz resonator $Q$ value. The quality factor decreased as the $\mathrm{pH}$ was increased at a fixed concentration. The quality factor dropped even more as the concentration was increased at constant $\mathrm{pH}$.

These results demonstrated that the buffer solution greatly affected the performance of the QCM, especially the quality factor. For example, the quality factor dropped from $936(0.01$ $\mathrm{M}, \mathrm{pH} 5.8)$ to $174(0.2 \mathrm{M}, \mathrm{pH} 8)$. The quality factor of a resonator is an important parameter that determines the frequency stability, and the conductance suggests the resonator vibration amplitude.

Understanding the mechanism of these effects will provide valuable guidance for the selection of the PB solution. The density and viscosity of these PB solutions are very close to that of pure water. Hence, the frequency shift induced by the liquid's mechanical properties should be several $\mathrm{Hz}$, which is much less than the experimental results (several $\mathrm{kHz}$ ). The difference may be caused by the liquid's electrical properties, especially its conductivity. Figure 3 shows a schematic diagram of the electric field distribution between the two electrodes of the QCM. ${ }^{(22)}$ 


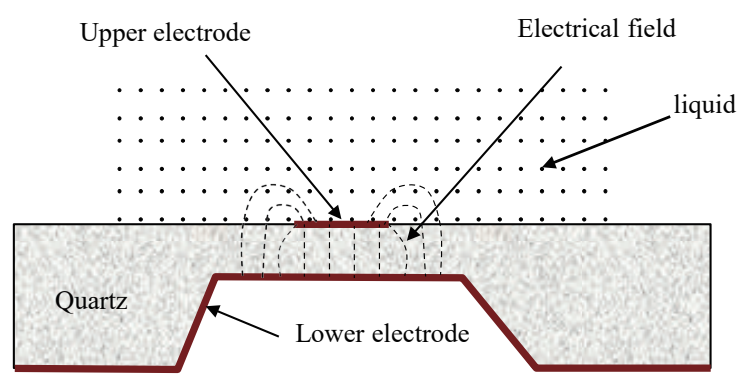

(a)

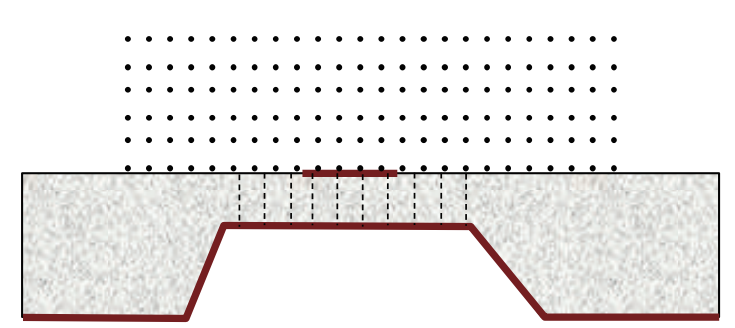

(b)

Fig. 3. (Color online) Schematic illustration of the electric field distribution of the QCM with an inverted-mesa structure: (a) in a nonconductive liquid and (b) in a perfectly conductive liquid.

The upper electrode is much smaller than the lower electrode, which causes the electrical field to penetrate into a liquid with a high dielectric constant, a phenomenon known as the enhanced stray effect, shown in Fig. 3(a). This effect can also explain the large frequency drop between in air and in water as shown in Fig. 1, because water has a higher relative dielectric constant (80) than air (1). When the liquid has a sufficiently high conductivity, the liquid itself can behave as an actual electrode, reducing the parasitic effect as shown in Fig. 3(b).

In this study, the PB solution has an intermediate conductivity; partial electrical field lines penetrate into the liquid. Because the dielectric constant change may be negligible for these concentrations of PB solutions used herein, any change in resonant characteristics should be caused by the conductivity. An increase in conductivity results in a decrease in resonant frequency as reported in Ref. 23. Furthermore, it can be easily understood that the conductivity would increase as the concentration of the $\mathrm{PB}$ solution was increased. For the same $\mathrm{PB}$ concentration, higher $\mathrm{pH}$ values indicate a higher $\mathrm{Na}_{2} \mathrm{HPO}_{4} / \mathrm{NaH}_{2} \mathrm{PO}_{4}$ ratio with a corresponding higher $\mathrm{Na}^{+}$concentration, which results in higher conductivity. That interpretation agrees well with the experimental results shown in Fig. 2(a). The $Q$ value also dropped with the increase in liquid conductivity, especially with the increase in $\mathrm{PB}$ concentration. In the low concentration range, the decrease in $Q$ value is considered the result of energy consumption by the conductive liquid, in which the electrical current is maintained and increased as the conductivity of the liquid increases. In the high concentration range, the electric lines leaking into the liquid diminish and the liquid itself acts as an electrical electrode, which extends the upper electrode area. This phenomenon practically altered the QCM electrode configuration, which equivalently caused the etched quartz area to be fully covered by the excitation electrodes. A fully covered electrode is not expected, and it may easily induce spurious vibrations leading to the decrease in $Q$ value.

In actual bio-sensing applications, $\mathrm{NaCl}$ is often added to the $\mathrm{PB}$ solution to increase the ionic strength. The addition of $\mathrm{NaCl}$ increases the conductivity of the solution. The relative effect on the change in resonant frequency and $Q$ value may also be demonstrated by adding $0.5 \%$ (wt.\%) $\mathrm{NaCl}$ into the $0.05 \mathrm{M}$ PB solution. Figure 4 shows the measured $Q$ value of the $0.05 \mathrm{M}$ $\mathrm{PB}$ solution with and without $\mathrm{NaCl}$. 


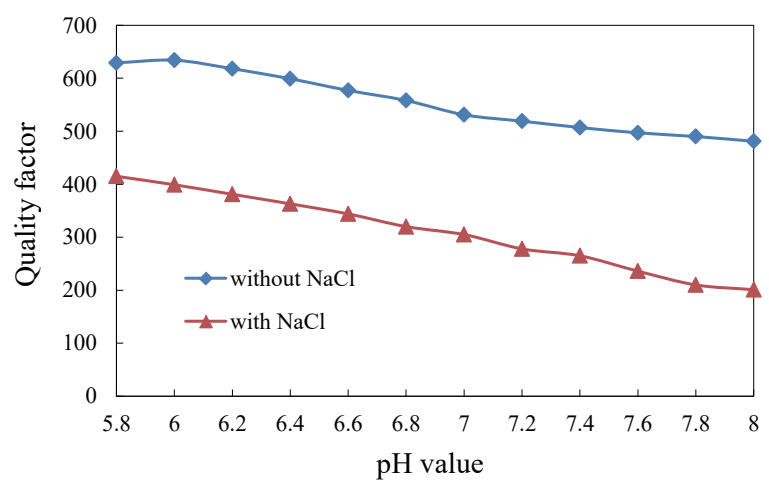

Fig. 4. (Color online) $Q$ value of a $0.05 \mathrm{M} \mathrm{PB}$ solution with and without added $\mathrm{NaCl}$.

The effects of $\mathrm{PB}$ concentration and $\mathrm{pH}$ value on the vibrational characteristics of a QCM with an inverted-mesa structure have been clearly demonstrated and considered with respect to solution conductivity. To avoid these effects, the lower electrode should be designed and fabricated to be smaller than the upper electrode.

\section{Conclusions}

When a QCM is used as a biosensor, its resonant characteristics are greatly affected by the buffer concentration and $\mathrm{pH}$ value when the upper electrode is smaller than the lower electrode. This study experimentally demonstrated that the resonant frequency and $Q$ value of the highfrequency QCM with an inverted-mesa structure decrease when the PB concentration and $\mathrm{pH}$ value are increased. The same trend is observed when $\mathrm{NaCl}$ is added to the solution. The decrease in the $Q$ value diminishes the resolution of the sensor. When this kind of QCM chip is used, the selection of buffer should be based on not only the bioreaction but also the QCM performance.

\section{Acknowledgments}

This work was financially supported by the Foundation of Key Laboratory of Micro-Inertial Instrument and Advanced Navigation Technology, Ministry of Education, China (201401), the Natural Science Foundation of Jiangsu Province (BK20171366), and the Fundamental Research Funds for the Central Universities (2242016K40041 and 2242017K1G009).

\section{References}

1 A. Salis and M. Monduzzi: Curr. Opin. Colloid Interface Sci. 23 (2016) 1.

2 A. Salis, D. Bilaničová, B. W. Ninham, and M. Monduzzi: J. Phys. Chem. B 111 (2007) 1149.

3 K. Tóth, E. Sedlák, M. Sprinzl, and G. Žoldák: Biochim. Biophys. Acta 1784 (2008) 789.

4 S. O. Ugwu and S. P. Apte: Pharm. Technol. 28 (2004) 86.

5 D. Roberts, R. Keeling, M. Tracka, C. F. van der Walle, S. Uddin, J. Warwicker, and R. Curtis: Mol. Pharmaceutics 12 (2015) 179. 
6 C. W. Chan and D. K. Smith: Supramol. Chem. 29 (2017) 688.

7 Z. Pei, H. Anderson, A. Myrskog, G. Dunér, B. Ingemarsson, and T. Aastrup: Anal. Biochem. 398 (2010) 161.

8 P. E. Vandeventer, J. S. Lin, T. J. Zwang, A. Nadim, M. S. Johal, and A. Niemz: J. Phys. Chem. B 116 (2012) 5661.

9 A. M. Michael, E. T. Joshua, and M. D. Gina: Biophys. Chem. 184 (2013) 29.

10 C. S. Weng, U. Hashim, and W. W. Liu: Proc. RSM2013 (IEEE, 2013) 200.

11 V. Volotovsky, A. P. Soldatkin, A. A. Shul'ga, V. K. Rossokhaty, V. I. Strikha, and A. V. El'skaya: Anal. Chim. Acta 322 (1996) 77.

12 G. Sauerbrey: Z. Phys. 155 (1959) 206.

13 T. Nomura and M. Okuhara: Anal. Chim. Acta 142 (1982) 281.

14 T. Nomura and T. Yanagihara: Anal. Chim. Acta 248 (1991) 329.

15 S. Bao, K. Fujio, and T. Nomura: Colloid Polym. Sci. 283 (2005) 619.

16 U. Hempel, R. Lucklum, P. R. Hauptmann, E. P. EerNisse, D. Puccio, and R. F. Diaz: Meas. Sci. Technol. 19 (2008) 055201.

17 W. Wang, C. Zhang, Y. Liu, and T. Ding: Sens. Actuators, B 156 (2011) 969.

18 T. Abe and H. Kato: Anal. Chem. 79 (2007) 6804.

19 Y. Hu, L. A. French Jr., K. Radecsky, M. P. da Cunha, P. Millard, and J. F. Vetelino: IEEE Trans. Ultrason. Ferroelectr. Freq. Control 51 (2004) 1373.

20 J. Liang, J. Huang, T. Zhang, J. Zhang, X. Li, and T. Ueda: Sensors 13 (2013) 12140.

21 J. Liang, J. Huang, S. Ding, and T. Ueda: Sens. Mater. 25 (2013) 519.

22 M. Rodahl, F. Höök, and B. Kasemo: Anal. Chem. 68 (1996) 2219.

23 M. Yang and M. Thompson: Anal. Chem. 65 (1993) 3591. 